\title{
XXVI Congress of the International Academy of Pathology
}

\author{
Hosted by United States - Canadian Academy of Pathology \\ September 16-21, 2006, Montreal, QC, Canada \\ Poster Presentations
}

\begin{abstract}
Autopsy
1 REVERSING THE SLOW DEATH OF THE CLINICAL POST MORTEM EXAMINATION: DEVELOPING THE POST OF THE PATHOLOGY LIAISON NURSE

Eileen Limacher, Norfolk and Norwich University Hospital, Norwich, United Kingdom; Urszula Carr, Addenbrooke's NHS Foundation Trust, Cambridge, United Kingdom; Lesley Bowker; Richard Ball, Norfolk and Norwich University Hospital, Norwich, United Kingdom

Background: By assessing the accuracy of clinical diagnosis and the effects of patient management, post mortem examinations (PMs) provide a valuable clinical audit tool. They also are important in teaching, training and research, and have benefits for surviving family members. Adult clinical (consented) PMs have seriously declined in recent decades, the decline in Norwich being approximately linear between 1996-2003. We wished to reverse this trend, not least to assist in training our medical students and junior histopathologists. We recognised that the burden on clinicians' time and their lack of training in obtaining consent for PM may have been contributing factors in decline of the clinical PM. In order to relieve the demand on doctors' time and to ensure that consent is properly elicited, the Trust established a post of Pathology Liaison Nurse (PLN). Here we describe how we established and developed the post of PLN and the current state of the adult clinical PM in Norwich.

Design: Developing the PLN post: the post was for a one-year trial period. The PLN was to ensure that family wishes about the extent of PM and the retention and disposal of material were properly carried out. The PLN received training in all aspects of the PM service and became confident in eliciting consent herself and in liasing with clinicians, pathologists and the bereavement service. The post was evaluated at the end of the trial year. The opinions of consultants and of some families were sought using questionnaires. These small surveys suggested that the PLN provided a valuable service, and the Trust Board retained the post. Review of the numbers and extent of consented post mortem examinations: The numbers of adult deaths in hospital and of clinical PMs were determined, allowing us to calculate the adult clinical PM rate (number of clinical PMs divided by the number of deaths. Results: The decline in the number of adult clinical PMs during $1996-2003$ was reversed during $2004-2005$. We undertook 167 adult clinical PMs in 1997, but there were only 34 in 2003, a reduction of nearly $80 \%$. The adult clinical PM rate fell from $8.4 \%$ in 1997 to $1.4 \%$ in 2003. During 2004, 45 adult clinical PMs were undertaken (clinical PM rate $=$ $1.8 \%$ ) with further improvement in 2005 (58 adult clinical PMs; clinical PM rate $=2.4 \%$ ). There have also been improvements in the extent of examination permitted and in the retention of material afterwards.

Conclusion: The decline in the adult clinical PM rate in Norwich was reversed in 2004 and 2005, coinciding with the appointment of the PLN and the internal publicity to promote her role and that of the clinical PM. The improvement has been modest, and the adult clinical PM rate remains far short of ideal. However, our achievement shows that the death of the adult clinical PM is not inevitable, and provides a good platform for further improvement. We believe that the profession should recognise its responsibility to improve the status of the clinical PM and act accordingly.
\end{abstract}

\section{Bone}

2 THE EXPERIMENTAL STUDY OF ACETABULAR BONE STRESS IN BIPOLAR HIP PROSTHESIS

Dan Nelu Anusca; Iancu Emil Plesea, University of Medicine and Pharmacy, Department of Orthopaedics, Craiova, Romania; Nicolae Iliescu, Polytechnics University, Department of Material Resistance, Bucuresti, Romania; Florin Poenaru, Emergency County University Hospital, Department of Orthopaedics, Craiova, Romania; Oltin Tiberiu Pop, University of Medicine and Pharmacy, Department of Pathology, Craiova, Romania; Stelian Danut Enache, Emergency County University Hospital, Department of Pathology, Craiova, Romania

Background: Although considered a revolutionary technique, hip arthroplasty didn't solve the problem of acetabular bone structures degenerative changes at the bone-prosthesis contact. These changes result in changing the bipolar prosthesis with a total hip prosthesis, metal-cup duo representing a temporal solution reserved for young people in order to preserve as much as possible the acetabular bone tissue. The purpose of this study is to identify the acetabular bone areas of maxim stress at the bone-duo prosthetic cup interface using experimental models.

Design: The materials consisted of a hipbone, a bipolar acetabular cup with outer diameter of $48 \mathrm{~mm}$, adequate for the hipbone and a standard neck with a $28 \mathrm{~mm}$ diameter prosthetic head. Postero-anterior and lateral radiographs of this joint model were made, illustrating two phases of the walking process. Serial axial CT scans (every $2 \mathrm{~mm}$ ) of the same articular model were made, from $3 \mathrm{~cm}$ above the acetabular dome to $1 \mathrm{~cm}$ below the inferior acetabular end. The obtained images were scanned and then a sufficient number of outline points were marked and related to a 3D coordinate system (XYZ). The finite elements model was obtained by computer aided assembling of the points resulted from the radiographic composing and the $\mathrm{CT}$ scans and further discretised by adding a number of 30000 points. Tensional and deformational status were assesed in all considered planes for all prosthesis components. A post-processing software traced the variation curves for the contact pressure at the components interface (hipbone-armour, armour-cup and cupfemoral head) using all the obtained data.

Results: The highest compressive tensions for the femoral prosthesis head were observed in the frontal plane and monopodalic support (ó $2=-176,46 \mathrm{MPa}$ ). The compressive tensions for the polyethylene cup are relatively small, the highest value also being in the frontal plane and monopodal support (ó $2=-15,83 \mathrm{MPa}$ ). The maximal tensions in the hipbone are observed also in the frontal plane and monopodal support in the proximity of the contact area with the prosthesis metallic armor ( $2=-70,43 \mathrm{MPa}$ ). In bipodal support, ó2 compressive tensions were very small for all the structures analyzed. The pressure variation curves show that maximal value for the contact pressure on the contact area between the armor and the hipbone was also recorded in the frontal plane and monopodal support $(\mathrm{pmax}=25 \mathrm{MPa}$ ). The maximal pressure in the sagital plane, on the same interface, in monopodal support, is much smaller (pmax $=8 \mathrm{MPa}$ ). For the same interface and bipodal support, in both planes, the maximal values of the contact pressure are much smaller (4 MPa and $3 \mathrm{MPa}$ respectively).

Conclusion: The obtained data suggestes that the main stress in the case of the prosthesis femoral head is in the frontal plane. The very high contact pressure recorded at the armorhipbone interface suggests a risk for bone tissue crush in certain areas of the contact surface. These destructions can lead, in time, to armor detachment from the bone cavity and thus requiring another surgical procedure.

3 MALIGNANCY IN GIANT CELL TUMORS OF BONE: IS THERE A REPRODUCIBLE HISTOLOGIC THRESHOLD? A STUDY OF THREE GIANT CELL TUMORS WITH WORRISOME FEATURES

Fadi Brimo, McGill University Health Centre, Montreal, QC, Canada; Mohamed Aziz; Gerald Rosen, St Vincents, Comprehensive Cancer Center, New York, NY, United States; Robert Turcotte; Ayoub Nahal, McGill University Health Centre, Montreal, QC, Canada Background: Giant cell tumor of bone is well known to recur and metastasize while preserving non atypical histology. Malignancy (MGCT), however, is a very rare event that has great implications for treatment. It occurs in $1 \%$ of all cases, typically following multiple recurrences and rarely de novo in a primary tumor. In the literature, the proposed criteria adopt the dedifferentiation theory and describe an undifferentiated sarcomatous overgrowth devoid of giant cells juxtaposed to a typical GCT, or in a previously diagnosed GCT. Atypia is generally accepted in GCT. However, cytologic and architectural atypia beyond the permissible level of benign GCT and short of malignancy can cause serious diagnostic dilemma as to its benign degenerative or malignant significance. Herein, we describe the histological features of three cases of GCT with such atypical foci in which the diagnosis of malignancy was controversial.

Design: Three cases of GCT with atypical foci derived from the Pathology files of the McGill University Health Center, Montreal, and Saint Vincent Comprehensive Cancer Centers, New York, were reviewed. The histologic features examined included: Stromal spindle cell overgrowth and hypercellularity, cytoatypia, hyperchromasia, presence/ absence of atypical mitotic figures, presence/absence of intermixed giant cells, and prominent nucleoli. Additional outside experts' opinions was sought given the complexity of the cases.

Results: One case presented with a solitary distal femoral mass. Two presented with lung metastases, 3 and 30 years following the initial diagnosis of GCT, respectively. The primary tumor in all three cases was treated by surgical curettage alone. The patient with the longer disease-free interval experienced two recurrences prior to metastases. All the three cases had somewhat similar histologic features: Typical GCT within which were foc of increased stromal cellularity, mild to moderate pleomorphism, rare atypical mitoses, and variably prominent nucleoli. In addition, one case showed a minute focus of stromal overgrowth of mildly atypical spindle cells with decreased number of giant cells. There was no hyperchromasia or frank high grade sarcomatous growth in any of the cases. Outside experts' opinions ranged from GCT, to GCT with areas suspicious for malignancy, to MGCT. The final diagnosis for which treatment was based upon was GCT (1 case) and 